\title{
Intracisternal Route of Administration
}

National Cancer Institute

\section{Source}

National Cancer Institute. Intracisternal Route of Administration. NCI Thesaurus. Code C38233.

Administration of a drug directly into the cerebrospinal fluid of the brain ventricles. It could be performed by direct injection into the cisterna magna or via permanently positioned tube. 